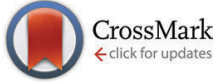

Cite this: Phys. Chem. Chem. Phys., 2015, 17, 7761

Received 28th January 2015, Accepted 17th February 2015

DOI: $10.1039 / c 5 c p 00532 a$

www.rsc.org/pccp

\title{
Boron nitride nanosheets as improved and reusable substrates for gold nanoparticles enabled surface enhanced Raman spectroscopy $\dagger$
}

\author{
Qiran Cai, ${ }^{a}$ Lu Hua Li, ${ }^{a}{ }^{a}$ Yuanlie Yu, ${ }^{b}$ Yun Liu, ${ }^{c}$ Shaoming Huang, ${ }^{d}$ Ying Chen, ${ }^{\text {a }}$ \\ Kenji Watanabe ${ }^{e}$ and Takashi Taniguchi ${ }^{e}$
}

\begin{abstract}
Atomically thin boron nitride (BN) nanosheets have been found to be excellent substrates for noble metal particles enabled surface enhanced Raman spectroscopy (SERS), thanks to their good adsorption of aromatic molecules, high thermal stability and weak Raman scattering. Faceted gold (Au) nanoparticles have been synthesized on BN nanosheets using a simple but controllable and reproducible sputtering and annealing method. The size and density of the Au particles can be controlled by sputtering time, current and annealing temperature etc. Under the same sputtering and annealing conditions, the Au particles on $B N$ of different thicknesses show various sizes because the surface diffusion coefficients of Au depend on the thickness of BN. Intriguingly, decorated with similar morphology and distribution of Au particles, BN nanosheets exhibit better Raman enhancements than silicon substrates as well as bulk BN crystals. Additionally, BN nanosheets show no noticeable SERS signal and hence cause no interference to the Raman signal of the analyte. The Au/BN substrates can be reused by heating in air to remove the adsorbed analyte without loss of SERS enhancement.
\end{abstract}

\section{Introduction}

Raman spectroscopy is a valuable non-destructive analytical tool for chemistry, biology, geology, solid-state physics as well as applications in pharmaceutical, cosmetic, food and environment-related industries. However, the very weak signal greatly limits the wide use of this technique. ${ }^{1}$ Surface enhanced Raman spectroscopy (SERS) which takes advantage of surface plasmons induced electromagnetic fields and/or chemical charge transfer can enormously increase the signals of Raman spectroscopy and therefore broaden its use, ${ }^{1-3}$ especially that the electromagnetic enhancement by metal nanoparticles can achieve single molecule detection. $^{4-6}$

\footnotetext{
${ }^{a}$ Institute for Frontier Materials, Deakin University, Geelong Waurn Ponds Campus, Waurn Ponds, VIC 3216, Australia. E-mail: luhua.li@deakin.edu.au, ian.chen@deakin.edu.au

${ }^{b}$ Advanced Membranes \& Porous Materials Center, King Abdullah University of Science \& Technology, Thuwal 23955-6900, Kingdom of Saudi Arabia

${ }^{c}$ Research School of Chemistry, The Australian National University, Canberra, ACT 0200, Australia

${ }^{d}$ Nanomaterials and Chemistry Key Laboratory, Wenzhou University, Wenzhou 325027, China

${ }^{e}$ National Institute for Materials Science, Namiki 1-1, Tsukuba, Ibaraki 305-0044, Japan

$\dagger$ Electronic supplementary information (ESI) available: Statistics of the diameter and height of the Au particles shown in Fig. 4a-c and SERS spectra of 1-3L BN. See DOI: $10.1039 / \mathrm{c} 5 \mathrm{cp} 00532 \mathrm{a}$
}

Two-dimensional (2D) nanomaterials, including graphene, hexagonal boron nitride (BN), molybdenum disulfide $\left(\mathrm{MoS}_{2}\right)$ nanosheets, provide new possibilities for SERS. Actually, graphene has been found to be an excellent substrate for SERS. It can not only boost Raman signals via chemical enhancement, ${ }^{7,8}$ but also attract analyte molecules in a more controllable way ${ }^{9}$ and quench fluorescence. ${ }^{10}$ However, chemical enhancement by graphene alone is not as effective as electromagnetic enhancement. Therefore, composites of graphene and metal nanoparticles have also been proposed for SERS to combine the advantages of graphene and the high enhancement factor from metal nanoparticles. ${ }^{9,11-13}$ In contrast, the possible use of non-carbon 2D nanomaterials in SERS has not been explored much. For example, there have been only a few reports of using $\mathrm{BN}$ nanosheets for Raman enhancement. ${ }^{8,14,15}$ Nevertheless, in spite of its similar crystal structure, BN has many different properties from the carbon counterpart, such as distinct chemical bonds and band structure, ${ }^{16}$ higher thermal and chemical stability, ${ }^{17}$ different surface state and weaker Raman scattering. ${ }^{18}$ These properties give BN certain advantages over carbon as a SERS substrate. For example, the stronger resistance to oxidation makes BN nanosheets more preferable for reusable SERS substrates than graphene, because $\mathrm{BN}$ nanosheets can sustain heating in air at higher temperatures to remove attached analytes for reuse. ${ }^{14}$ In addition, BN nanosheets only show a Raman $\mathrm{G}$ band of low intensity, ${ }^{17-19}$ which is too weak to show in the SERS spectrum and therefore only Raman signals of analyte are present. 
Here, we report that atomically thin $\mathrm{BN}$ nanosheets are excellent substrates for metal nanoparticles enabled SERS. Gold $\mathrm{Au}$ ) nanoparticles were prepared on $\mathrm{BN}$ by a straightforward but effective and repeatable sputtering and annealing method. The size of the metal particles can be controlled by the thickness of the sputtered thin film. Rhodamine 6G (R6G) solution was used to compare the Raman enhancements of $\mathrm{Au}$ decorated silicon oxide, atomically thin $\mathrm{BN}$ and bulk hBN particles. Interestingly, the atomically thin BN substrate showed the strongest Raman signals of R6G. This phenomenon can be attributed to the unique properties of BN nanosheets. Furthermore, the Au nanoparticles decorated BN substrates for SERS are reusable with no noticeable decrease of Raman enhancement.

\section{Experimental}

\section{Preparation of BN nanosheets}

The BN nanosheets were exfoliated from high-quality hBN single crystals $^{20,21}$ on silicon wafer covered by $90 \mathrm{~nm}$ thick silicon oxide $\left(\mathrm{SiO}_{2} / \mathrm{Si}\right)$ using the Scotch tape technique. ${ }^{17,19}$ Heat treatments at $350{ }^{\circ} \mathrm{C}$ were conducted in a tubular furnace in air for $3 \mathrm{~h}$ to remove possible moisture and adhesive residue on the samples. The nanosheets were identified using an Olympus BX51 optical microscope equipped with a DP71 camera. Then, a Cypher atomic force microscope (AFM) was used to measure the nanosheet thickness in tapping and contact modes using $\mathrm{Si}$ cantilevers. The nanosheets were also analyzed using a Renishaw inVia Raman microscope equipped with a $514.5 \mathrm{~nm}$ laser.

\section{Synthesis of $\mathrm{Au}$ particles}

Thin layers of $\mathrm{Au}$ film ( 8-12 $\mathrm{nm}$ in thickness) were firstly sputtered on the $\mathrm{SiO}_{2} / \mathrm{Si}$ substrate with the $\mathrm{BN}$ nanosheets and bulk hBN particles under the protection of argon (Ar) at a pressure of $\sim 0.5 \times 10^{-1} \mathrm{mbar}$ (SCD050, Bel-Tec). The sputtering current was $40 \mathrm{~mA}$ and the sputtering time was in the range of 20 to $40 \mathrm{~s}$. Then, the Au film covered substrates were annealed at $600{ }^{\circ} \mathrm{C}$ for $1 \mathrm{~h}$ in an $\mathrm{Ar}$ atmosphere. The size of the Au particles was measured using the AFM.

\section{SERS measurements}

The Au particles covered substrates were immersed in $10^{-6} \mathrm{M}$ R6G ( $\geq 95 \%$, Fluka) water (Milli-Q) solution for $1 \mathrm{~h}$, followed by washing with Milli-Q water to remove remaining drops of R6G solution. The reusability was tested by heating the substrate with $\mathrm{R} 6 \mathrm{G}$ at $400{ }^{\circ} \mathrm{C}$ in air for $5 \mathrm{~min}$ and re-immersed in the R6G solution of the same concentration up to five times. A $100 \times$ objective lens with a numerical aperture of 0.90 was used in the Raman measurements. The laser power was $\sim 2.5 \mathrm{~mW}$. All Raman spectra were calibrated with the Raman band of Si at $520.5 \mathrm{~cm}^{-1}$.

\section{Results and discussion}

The $\mathrm{BN}$ nanosheets exfoliated on $\mathrm{SiO}_{2} / \mathrm{Si}$ substrates were heated at $350{ }^{\circ} \mathrm{C}$ in air for $3 \mathrm{~h}$ to remove possible moisture and tape residue. The moisture may affect the AFM measurements of the thickness of the nanosheets; the tape residue could weaken the adsorption capability of BN nanosheets as well as cause extraneous Raman signals. Fig. 1a shows an optical photograph of BN nanosheets of 1-3 layers (L) and a bulk hBN after heating: the thinner the nanosheets, the lower the optical contrast. Consistent to our previous reports, ${ }^{17,19}$ the Raman frequency of the $\mathrm{G}$ band of atomically thin $\mathrm{BN}$ on the $\mathrm{SiO}_{2} / \mathrm{Si}$ substrate upshifts with the decrease of thickness: $1370.5 \mathrm{~cm}^{-1}$ for $1 \mathrm{~L}, 1370.0 \mathrm{~cm}^{-1}$ for $2 \mathrm{~L}$, $1368.1 \mathrm{~cm}^{-1}$ for $3 \mathrm{~L}$ and $1366.8 \mathrm{~cm}^{-1}$ for the bulk. In addition, thinner nanosheets generally show broader G bands. AFM results in Fig. 1c and d show that the thicknesses of the 1-3L BN are 0.40, 0.85 and $1.25 \mathrm{~nm}$, respectively.

$\mathrm{Au}$ nanoparticles were produced on the $\mathrm{BN}$ and $\mathrm{SiO}_{2} / \mathrm{Si}$ substrate by a sputtering and annealing method. A thin layer of $\mathrm{Au}$ film was deposited on the substrate by sputter coating and then the annealing at $600{ }^{\circ} \mathrm{C}$ transferred the film to $\mathrm{Au}$ particles. Few-layer $\mathrm{BN}$ is visible under an optical microscope after sputtering and annealing (Fig. 1e and f). It is well-known that surface plasmon resonance and therefore SERS enhancement are
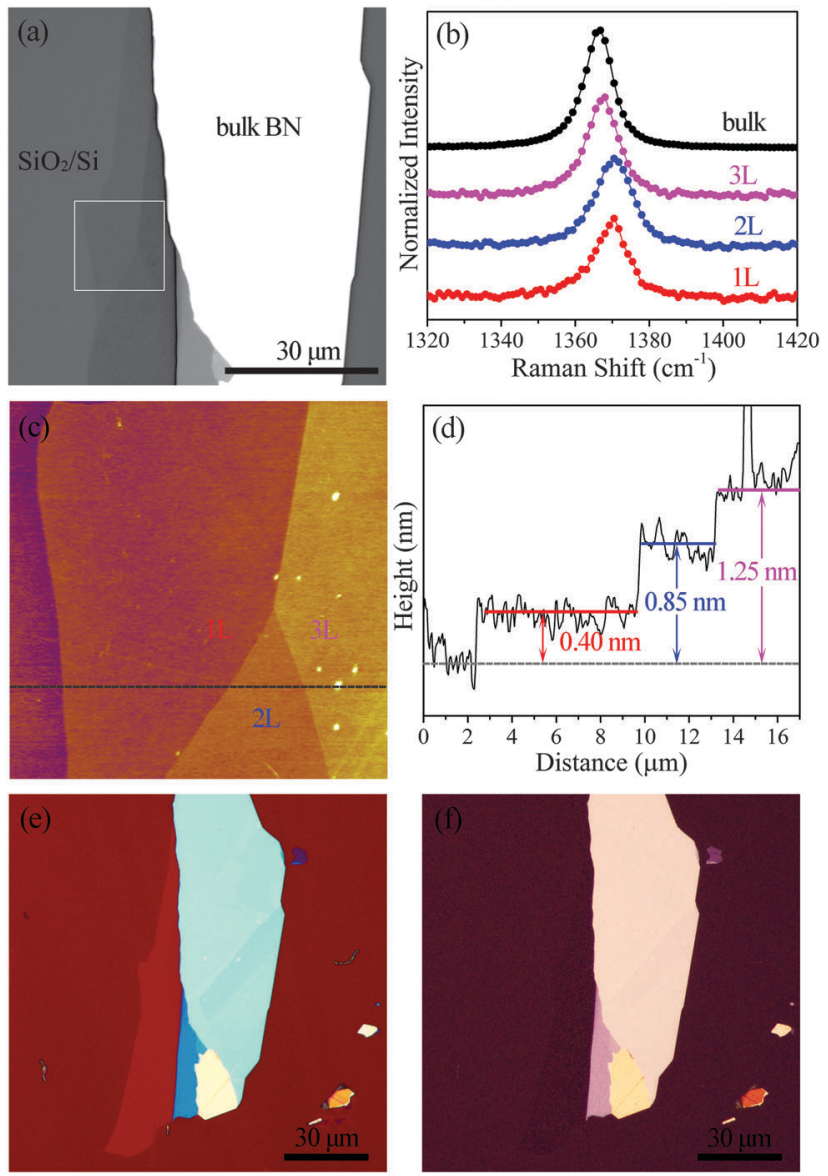

Fig. 1 (a) Optical image of 1-3L BN nanosheets along with a bulk BN on $\mathrm{SiO}_{2} / \mathrm{Si}$ substrates; (b) normalized Raman spectra of the $\mathrm{BN}$ of different thicknesses; (c) AFM image of the 1-3L BN, as shown in the square in (a); (d) height trace of the dashed line in the AFM image in (c); (e) optical image of the few-layer BN covered by a thin film of sputtered Au; (f) optical image of the same area after annealing to transfer the Au film to particles. 

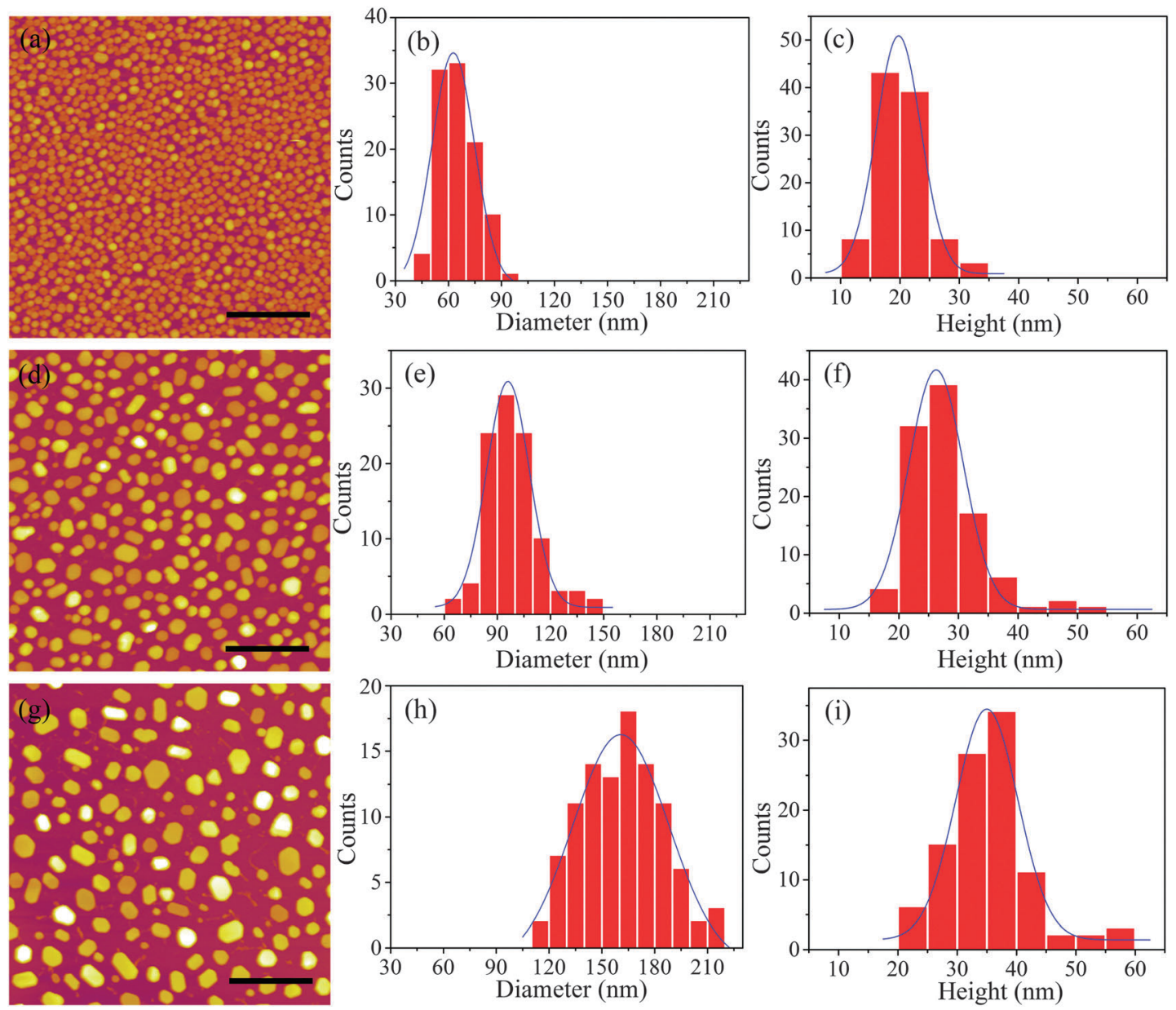

Fig. 2 (a), (d) and (g) AFM images showing the size and distribution of Au particles on BN using different sputtering times (20, 30 and 40 s, respectively); (b), (e) and (h) statistics of the AFM-derived particle diameter and corresponding Gaussian fits of the distribution; (c), (f) and (i) statistics of particle height and corresponding fits. All scale bars are $500 \mathrm{~nm}$.

highly dependent on the size, shape and distribution of metal nanoparticles. It is found that the size and distribution of $\mathrm{Au}$ particles on BN can be easily controlled by this synthesis method. The AFM images in Fig. 2a, d and g demonstrate the effect of sputtering time (namely, 20,30 and $40 \mathrm{~s}$ ) on the size and distribution of Au particles on BN; while other conditions, such as the sputtering current $(40 \mathrm{~mA})$ and annealing temperature $\left(600{ }^{\circ} \mathrm{C}\right)$, are kept the same. According to AFM measurements, the thicknesses of the Au films from the three sputtering time are about 8,10 and $12 \mathrm{~nm}$, respectively. With the increase of sputtering time, i.e. deposition thickness, the Au particles become larger and hence lower in density. The average diameter is $63 \mathrm{~nm}$ for the sputtering time of $20 \mathrm{~s}, 94 \mathrm{~nm}$ for $30 \mathrm{~s}$ and $154 \mathrm{~nm}$ for $40 \mathrm{~s}$, respectively (Fig. 2b, e and h). The change in particle height follows a similar trend: average $20 \mathrm{~nm}$ for the sputtering time of $20 \mathrm{~s}$, $27 \mathrm{~nm}$ for $30 \mathrm{~s}$ and $35 \mathrm{~nm}$ for $40 \mathrm{~s}$, respectively (Fig. 2c, f and i). Note that the uniformity of both particle diameter and height decreases for longer sputtering time, i.e. broader distributions in Fig. 2e, $\mathrm{f}, \mathrm{h}$ and $\mathrm{i}$ than those in Fig. $2 \mathrm{~b}$ and $\mathrm{c}$.

Most of the Au particles have a hexagon shape and flat top, suggesting that Au particles are highly crystallized and with a preferential orientation of the (111) planes parallel to the substrate surface. ${ }^{13,22,23}$ This suggests that the Au films melt (at least partially) at the annealing temperature of $600{ }^{\circ} \mathrm{C}$ (the melting point of Au could be strongly influenced by its size or thickness $)^{24}$ and recrystallized during the cooling process. The different stress and crack in the Au films of different thicknesses during the heating can explain the different particle size and distribution achieved by different sputtering time. The formation of larger particles from the thicker Au film produced by longer sputtering time is due to the tendency of thicker film to break into larger fractions during heating. The less uniformity in particle size and distribution from the longer sputtering time might be attributed to less homogeneity in fractions of thicker film during heating. It should be mentioned that the size and distribution of Au particles can also be adjusted by sputtering current and annealing temperature, which is not shown here.

It is also found that under the same sputtering and annealing conditions, the size and distribution of Au particles are slightly different among atomically thin $\mathrm{BN}$ nanosheets, bulk BN and $\mathrm{SiO}_{2} / \mathrm{Si}$ wafer. The Au particles on $\mathrm{BN}$ are generally larger than those on the $\mathrm{SiO}_{2} / \mathrm{Si}$ wafer, but few-layer BN had smaller particles than bulk BN (Fig. 3). The particle density of the three substrates follows a contrary trend. The differences are due to a kinetic 

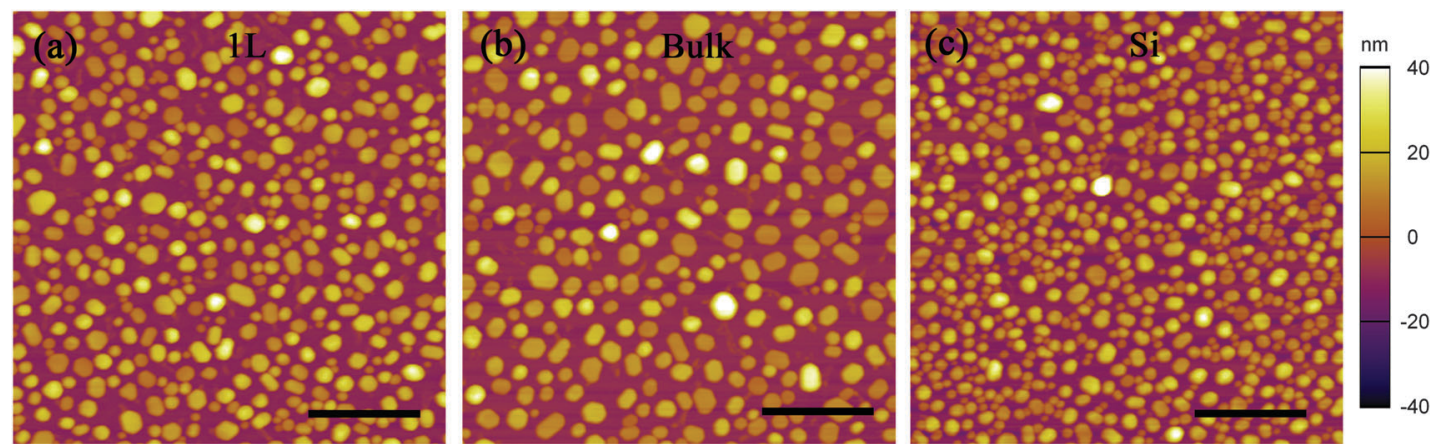

Fig. 3 AFM images of Au particles produced on (a) $1 \mathrm{~L} \mathrm{BN}$, (b) bulk BN and (c) $\mathrm{SiO}_{2} / \mathrm{Si}$ wafer by $30 \mathrm{~s}$ sputtering and $600{ }^{\circ} \mathrm{C}$ annealing. The scale bars are $500 \mathrm{~nm}$.

factor: surface diffusion. As mentioned previously, the Au films melt and hence can diffuse on the substrates during the annealing at $600{ }^{\circ} \mathrm{C}$. Therefore, the surface diffusion coefficient, i.e. the rate at which Au migrates and integrates with surrounding clusters to form larger particles, determines the size of the synthesized $\mathrm{Au}$ particles. A higher surface diffusion coefficient means a larger migrating rate and hence higher probability to form larger $\mathrm{Au}$ particles, and vice versa. ${ }^{25}$ It is not surprising that the diffusion coefficient of $\mathrm{BN}$ is larger than $\mathrm{SiO}_{2} / \mathrm{Si}^{26}$ but this is the first observation that few-layer and bulk BN have different diffusion coefficients. The difference in diffusion should be caused by different flatness. ${ }^{27}$ Atomically thin nanosheets are flexible and tend to follow the roughness of the substrate, normally resulting in larger roughness than the bulk crystal. ${ }^{28,29}$ The roughness of $1 \mathrm{~L}$ BN can reduce the diffusion coefficient and result in smaller Au particles.
The size and distribution of Au particles were optimized for SERS purpose. Because the produced Au particles are slightly different for $\mathrm{BN}$ nanosheets, bulk $\mathrm{BN}$ and $\mathrm{SiO}_{2} / \mathrm{Si}$ wafer under the same sputtering and annealing conditions, sputtering time was tuned to obtain similar size and distribution of Au particles on the three substrates for direct comparison of their SERS enhancements. As shown by the AFM images in Fig. $4 a-c, A u$ particles of similar morphology and density were formed on $1 \mathrm{~L}$ $\mathrm{BN}$, bulk BN and $\mathrm{SiO}_{2} / \mathrm{Si}$ wafer at sputtering time of 32,30 and $35 \mathrm{~s}$, respectively. The statistics of the diameter and height of the $\mathrm{Au}$ particles on the three substrates can be found in the ESI $\dagger$ (Fig. S1). The Raman enhancements of the three $\mathrm{Au}$ particles decorated substrates are compared using $10^{-6} \mathrm{M}$ aqueous solution of R6G (Fig. 4d). Raman signals of R6G were greatly enhanced by all three substrates, indicating that the faceted Au particles produced by the sputtering and annealing
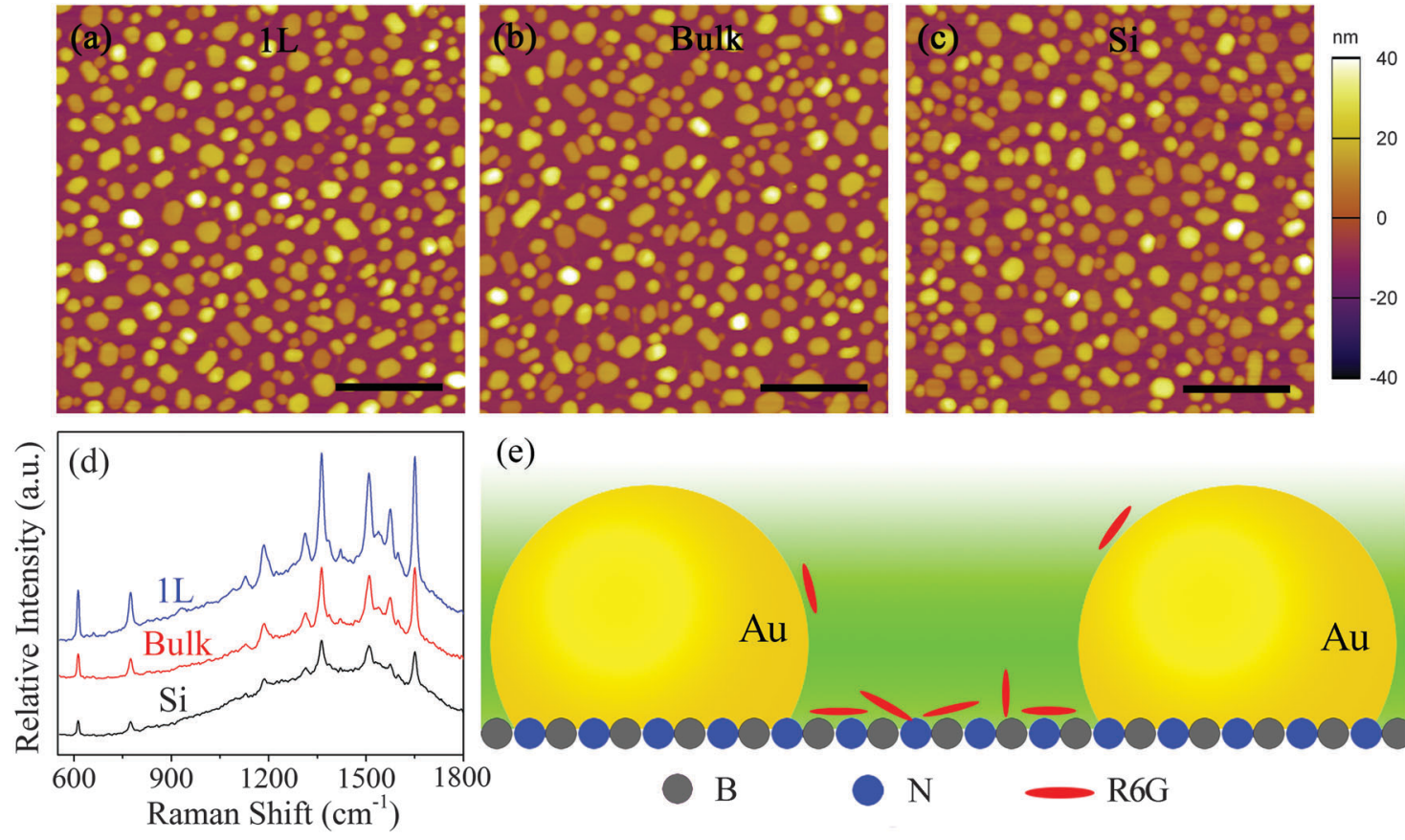

(e)

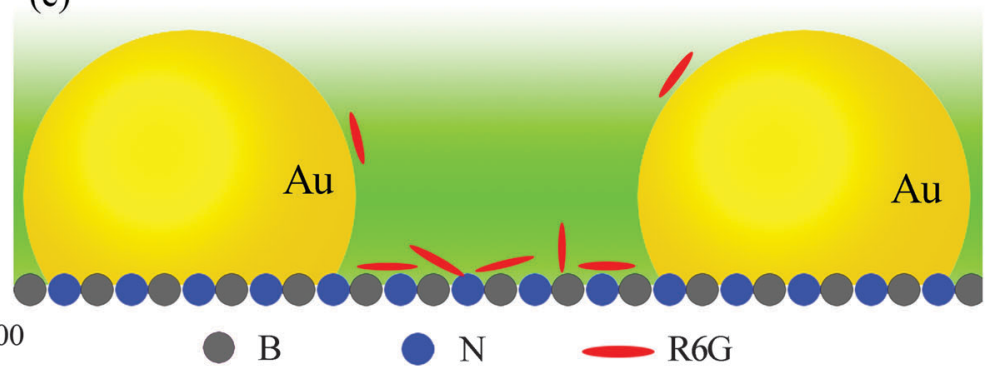

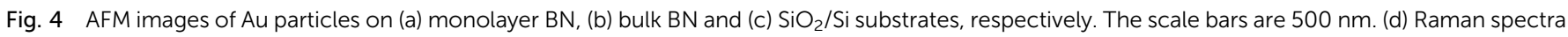

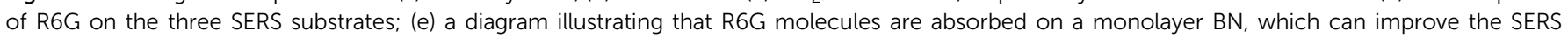
enhancement. 
method are suitable for SERS applications. However, both $1 \mathrm{~L}$ and bulk BN show more signal enhancements than the $\mathrm{SiO}_{2} / \mathrm{Si}$ substrate, mainly because $\mathrm{BN}$ is better in adsorption of R6G via $\pi-\pi$ interactions. As illustrated in Fig. 4e, although Au particles are able to adsorb a certain amount of R6G molecules, the surface of BN can adsorb dramatically more R6G than $\mathrm{SiO}_{2} / \mathrm{Si}$. These additionally adsorbed molecules are located in the so-called "hot spots" or gaps among Au particles and hence greatly electromagnetically enhanced, leading to the stronger Raman signals. The dipole interactions between $\mathrm{BN}$ and R6G may also contribute to the better enhancement. ${ }^{8}$

Intriguingly, we noticed that atomically thin BN (including 1-3L) shows more intensified R6G Raman signals than bulk BN particles (Fig. 4d and Fig. S2 in the ESI $\dagger$ ). This phenomenon should not be caused by the dipole interaction induced chemical enhancement, because the dipole interactions among BN of different thicknesses should be similar, as demonstrated in a previous study which shows that $\mathrm{BN}$ of different thicknesses had the same magnitude of chemical enhancement in Raman. ${ }^{8}$ Therefore, it could be due to stronger adsorption capability of atomically thin $\mathrm{BN}$ than bulk BN. This special property of $\mathrm{BN}$ nanosheets needs further study and may also be applicable to graphene and other 2D nanomaterials.

It should also be emphasized that different from graphene which normally introduces strong intrinsic Raman bands of carbon (G, 2D and possibly D bands) to the SERS spectrum, ${ }^{10,30-32}$ the Raman signal of BN nanosheets seems to be barely enhanced by the Au particles and is absent from the SERS results (black spectrum labelled "after heating" in Fig. 5). This makes BN more attractive for SERS, because it does not cause interference with analyte signals.

The reusability of the Au particles coated $1 \mathrm{~L} \mathrm{BN}$ was also tested. To clean off the adsorbed R6G molecules, the substrate

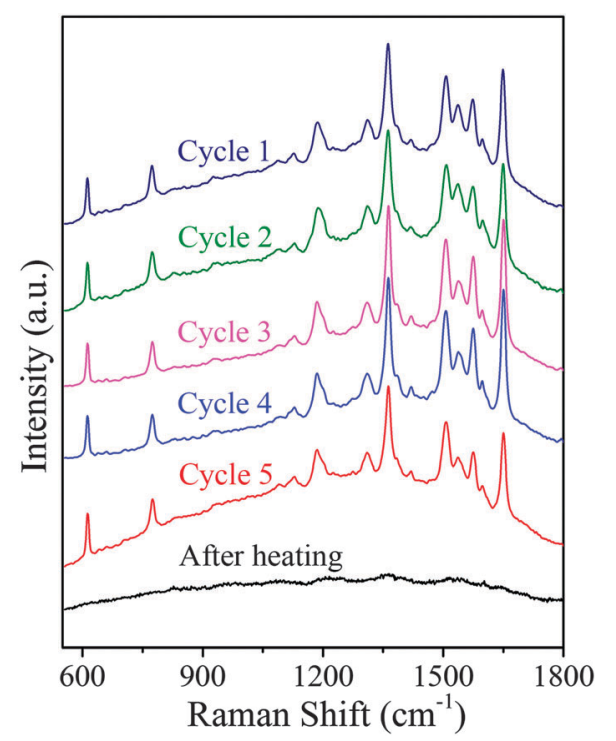

Fig. 5 Raman spectra of R6G $\left(10^{-6} \mathrm{M}\right)$ on a SERS substrate of Au decorated $1 \mathrm{~L} \mathrm{BN}$ after five cycles of the reusability test. A typical Raman spectrum after heating at $400{ }^{\circ} \mathrm{C}$ to remove the adsorbed R6G is also shown for comparison. All the spectra were acquired under the same conditions. was heated at $400{ }^{\circ} \mathrm{C}$ in air for $5 \mathrm{~min}$. After the heating treatment, almost no Raman signal of R6G was observed and there was only a featureless fluorescence background (black, Fig. 5), implying effective removal of the R6G. The heated substrate was then re-immersed in R6G solution of the same concentration for the SERS test, as described previously. The heating and re-adsorption processes were repeated five times on the same 1L BN decorated by Au particles. Fig. 5 shows the Raman spectra from the five cycles of the reusability test. It is worth emphasizing that the $1 \mathrm{~L} \mathrm{BN}$ shows no noticeable loss of SERS enhancement after the five cycles, as there is almost no change in the Raman intensity and the spectral features and peak positions of R6G. The good recyclability can be attributed to the excellent thermal stability of atomically thin BN: monolayer BN can sustain more than $800{ }^{\circ} \mathrm{C}$ in air; while graphene starts oxidation at less than $300{ }^{\circ} \mathrm{C} .{ }^{14,17}$ Thus, the Au/BN hybrid enables reusable SERS substrates that can withstand multiple thermal regeneration cycles.

\section{Conclusions}

In summary, BN nanosheets were used as substrates for highlysensitive metal nanoparticles enabled SERS substrates. The Au particles were produced by a simple but effective sputtering and annealing method. The particle size and distribution can be controlled by sputtering current, time and annealing temperature, and hence optimised for SERS. It is found that BN nanosheets show better Raman enhancements than bulk BN and $\mathrm{SiO}_{2} / \mathrm{Si}$, no interference with Raman signals from the analyte and a good reusability without noticeable loss of enhancement. Therefore, BN nanosheets are excellent substrates for metal particles enabled SERS.

\section{Acknowledgements}

L. H. Li thanks the financial support from ADPRF2014 and CRGS2015. Y. Chen thanks the funding from the Australian Research Council under the Discovery Program.

\section{Notes and references}

1 A. Campion and P. Kambhampati, Chem. Soc. Rev., 1998, 27, 241-250.

2 G. A. Baker and D. S. Moore, Anal. Bioanal. Chem., 2005, 382, 1751-1770.

3 C. L. Haynes, C. R. Yonzon, X. Zhang and R. P. Van Duyne, J. Raman Spectrosc., 2005, 36, 471-484.

4 S. Nie and S. R. Emory, Science, 1997, 275, 1102-1106.

5 A. M. Michaels, J. Jiang and L. Brus, J. Phys. Chem. B, 2000, 104, 11965-11971.

6 H. Xu, E. J. Bjerneld, M. Käll and L. Börjesson, Phys. Rev. Lett., 1999, 83, 4357-4360.

7 X. Ling, L. Xie, Y. Fang, H. Xu, H. Zhang, J. Kong, M. S. Dresselhaus, J. Zhang and Z. Liu, Nano Lett., 2010, 10, 553-561. 
8 X. Ling, W. Fang, Y.-H. Lee, P. T. Araujo, X. Zhang, J. F. Rodriguez-Nieva, Y. Lin, J. Zhang, J. Kong and M. S. Dresselhaus, Nano Lett., 2014, 14, 3033-3040.

9 G. Lu, H. Li, C. Liusman, Z. Yin, S. Wu and H. Zhang, Chem. Sci., 2011, 2, 1817-1821.

10 Y. Wang, Z. Ni, H. Hu, Y. Hao, C. P. Wong, T. Yu, J. T. Thong and Z. X. Shen, Appl. Phys. Lett., 2010, 97, 163111.

11 W. Xu, X. Ling, J. Xiao, M. S. Dresselhaus, J. Kong, H. Xu, Z. Liu and J. Zhang, Proc. Natl. Acad. Sci. U. S. A., 2012, 109, 9281-9286.

12 Y.-C. Liu, C.-C. Yu and S.-F. Sheu, J. Mater. Chem., 2006, 16, 3546-3551.

13 H. Zhou, F. Yu, M. Chen, C. Qiu, H. Yang, G. Wang, T. Yu and L. Sun, Carbon, 2013, 52, 379-387.

14 Y. Lin, C. E. Bunker, K. S. Fernando and J. W. Connell, ACS Appl. Mater. Interfaces, 2012, 4, 1110-1117.

15 S. S. Yang, Z. C. Zhang, J. Zhao and H. L. Zheng, J. Alloys Compd., 2014, 583, 231-236.

16 N. Ooi, A. Rairkar, L. Lindsley and J. B. Adams, J. Phys.: Condens. Matter, 2006, 18, 97-115.

17 L. H. Li, J. Cervenka, K. Watanabe, T. Taniguchi and Y. Chen, ACS Nano, 2014, 8, 1457-1462.

18 R. V. Gorbachev, I. Riaz, R. R. Nair, R. Jalil, L. Britnell, B. D. Belle, E. W. Hill, K. S. Novoselov, K. Watanabe, T. Taniguchi, A. K. Geim and P. Blake, Small, 2011, 7, 465-468.

19 L. H. Li, E. J. G. Santos, T. Xing, E. Cappelluti, R. Roldán, Y. Chen, K. Watanabe and T. Taniguchi, Nano Lett., 2015, 15, 218-223.
20 T. Taniguchi and K. Watanabe, J. Cryst. Growth, 2007, 303, 525-529.

21 L. Li, L. H. Li, Y. Chen, X. J. Dai, P. R. Lamb, B. M. Cheng, M. Y. Lin and X. W. Liu, Angew. Chem., Int. Ed., 2013, 52, 4212-4216.

22 Y. Golan, L. Margulis and I. Rubinstein, Surf. Sci., 1992, 264, 312-326.

23 T. Karakouz, A. B. Tesler, T. A. Bendikov, A. Vaskevich and I. Rubinstein, Adv. Mater., 2008, 20, 3893-3899.

24 P. Buffat and J. P. Borel, Phys. Rev. A: At., Mol., Opt. Phys., 1976, 13, 2287.

25 H. Zhou, C. Qiu, Z. Liu, H. Yang, L. Hu, J. Liu, H. Yang, C. Gu and L. Sun, J. Am. Chem. Soc., 2009, 132, 944-946.

26 F. P. Chiaramonte and B. N. Rosenthal, J. Am. Ceram. Soc., 1991, 74, 658-661.

27 Y. W. Koh and S. Manzhos, MRS Commun., 2013, 3, 171-175.

28 M. Ishigami, J. Chen, W. Cullen, M. Fuhrer and E. Williams, Nano Lett., 2007, 7, 1643-1648.

29 U. Stöberl, U. Wurstbauer, W. Wegscheider, D. Weiss and J. Eroms, Appl. Phys. Lett., 2008, 93, 051906.

30 C. Xu and X. Wang, Small, 2009, 5, 2212-2217.

31 X. Liu, L. Cao, W. Song, K. Ai and L. Lu, ACS Appl. Mater. Interfaces, 2011, 3, 2944-2952.

32 L. Polavarapu, K. K. Manga, K. Yu, P. K. Ang, H. D. Cao, J. Balapanuru, K. P. Loh and Q.-H. Xu, Nanoscale, 2011, 3, 2268-2274. 